\title{
Alignment of Selected Veterinary Education Competencies With the Interprofessional Professionalism Assessment
}

\author{
John H. Tegzes ${ }^{1 *}$ and Jody S. Frost ${ }^{2}$ \\ ${ }^{1}$ Office of Mission Integration, College of Veterinary Medicine, Western University of Health Sciences, Pomona, CA, \\ United States, ${ }^{2}$ National Academies of Practice, Lusby, MD, United States
}

Keywords: interprofessional, professionalism, competency-based education, assessment, Entrustable Professional Activities (EPAs)

\section{INTRODUCTION}

The move toward outcomes and competency-based veterinary education began decades ago but has accelerated in the past few years. While many advances in curriculum structure and delivery are replete in the literature, assessment methods remain challenging and less explored. This is especially true for competency domains that are behavior-based such as professionalism, which has been challenging to merely define let alone assess. The Interprofessionalism Professionalism

OPEN ACCESS

Edited by: Jared Andrew Danielson, lowa State University, United States

Reviewed by: Melinda Ann Frye, Colorado State University,

United States

*Correspondence:

John H. Tegzes

jtegzes@westernu.edu

Specialty section:

This article was submitted to Veterinary Humanities and Social

Sciences,

a section of the journa

Frontiers in Veterinary Science

Received: 31 March 2021

Accepted: 10 June 2021

Published: 09 July 2021

Citation:

Tegzes JH and Frost JS (2021) Alignment of Selected Veterinary Education Competencies With the Interprofessional Professionalism
Assessment (IPA) is a validated tool that is used in veterinary education and is useful in assessing competency domains that have remained challenging for educators. Here we present the challenges and solutions for assessing competency in veterinary education and provide examples and data from tools that may be useful especially in areas such as professionalism.

\section{BACKGROUND}

It is important to consider the pathway that veterinary education has traveled toward competency-based education and assessment before diving into specific assessment methods. This section will provide a brief overview of the history of outcomes-based and competency-based education as it applies to veterinary education.

\section{Outcomes-Based Education}

Outcomes-based education was initiated in 1949 when educational psychologist Ralph Tyler presented four questions to educational institutions now known as the "Tyler rationale" $(1,2)$. At the time, the questions were a sharp contrast to how educators organized or conceptualized their work. Through these questions, he ushered in the idea that education should be driven by outcomes, asking:

"1 What educational purposes should the school seek to attain?

2 How can learning experiences be selected which are likely to be useful in attaining these objectives?

3 How can learning experiences be organized for effective instruction?

4 How can the effectiveness of learning experiences be evaluated?"

These questions have led to subsequent frameworks to evaluate teaching and learning. Educational researchers have added wisdom over the years. Many have been adopted as a matter of routine in contemporary education, whether in primary or in health professions education. Educators commonly use Bloom's taxonomy for describing the progression of learning in three domains: 
cognitive, psychomotor, and affective (3). Kirkpatrick's framework is often used to measure the effectiveness of curricular methods (4). Based on these frameworks, it might seem predictable that the next evolution in education would be teaching for competence.

\section{Competency-Based Education}

Competency-based education embraces outcomes-based learning, advancing the framework to another level, especially when applied to healthcare professions' education. It is organized around competencies, or specific abilities, as the outcomes of the curriculum (5). In 1978, McGaghie et al. (5) described two alternative curriculum models in medical education that could replace the widely accepted subject-centered structure of most curricula of the time. The authors described the first as an integrated program where learning and teaching fuse formerly separate medical disciplines by using organ systems or medical problems as the organizing structure. The second model described was one that focuses on the functional elements of medical practice where the emphasis is on learning how to practice medicine as opposed to accumulating knowledge about medical practice. The authors called this "competency-based" because the emphasis was on learning how to practice medicine rather than on the accumulation of knowledge $(6,7)$.

This evolution from educating for knowledge to educating for competency seems logical, but also poses many questions without necessarily obvious answers. For instance, what is meant by competence? How can it be measured? Rather than highlighting a specific skill or procedure, competence is measured by comprehensive performing. Caring for a patient involves more than a specific skill or even set of skills. It requires knowledge, clinical expertise, and human connectedness demonstrated as behaviors (7). As competency-based education has been defined it leads to a primary focus of education on the desired outcomes for learners rather than on the structure of individual courses (8). Competence is an amalgamation of taking what was learned in the classroom, what was learned through independent study and reading, practicing specific clinical skills, communicating, working in a team, and finally reflecting upon all of these elements and modifying performance. Integrating all of these components within a specific context enables one to practice a specific healthcare profession (7). While at first glance it might seem like a practicing physician, dentist, or veterinarian might need to demonstrate competence while caring for many different types of patients with various diseases, the competency frameworks are quite short and non-specific, making it all the more difficult to assess for competency. Not surprisingly it would explain why educators may struggle with formulating specific assessments, and why sometimes you may hear the phrase, "I know competence when I see it" before being able to provide an explicit definition.

\section{Competency-Based Frameworks}

In Canada, frameworks for identifying and defining competence in medical education were initially sparked by calls in the 1990s for physician accountability and professionalism (7). CanMEDS defined a framework of competencies designed to address the roles physicians have in meeting societal needs. The CanMEDS Framework identified the competencies of physicians, which included medical expert, communicator, collaborator, manager, health advocate, scholar, and professional (7). Today there are similar frameworks in the United States, the United Kingdom, and in The Netherlands $(2,6)$. According to ten Cate (2) these competency frameworks contain logical sets of qualities that every physician should acquire and are still a theoretical construct. The defined competencies are general attributes of a good doctor. Unfortunately, as we begin to assess competencies in educational settings, they tend to get reduced to a detailed list of skills. Often the skills most emphasized are those that are procedural with a specific endpoint that can be measured for success (i.e., inserting a thoracostomy tube). Educators tend to focus on skills and activities that are objective, measurable, and repeatable (reproducible). This creates dissent and debate among educators when competencies have titles such as collaborator and professional which are often measured somewhat subjectively and contextually in the clinical settings.

As competency frameworks in medical education have been adopted across various countries, so too have they spread to other professions (9-12). The existing competency frameworks cannot simply be applied to the profession of veterinary medicine without revision and adaptation. While educating physicians and veterinarians share many similarities, there are also clear differences. For instance, most physicians must undergo further post-graduate education in the form of residency programs before becoming licensed for practice. Most veterinary students must be practice-ready on day 1 following graduation, with residency and fellowship programs remaining optional, and occurring after full licensure. Therefore, their readiness for practice upon graduation is paramount and different. Much work has been done to establish a competency-based framework for veterinary education. In The Netherlands, the work of Bok et al. paved the early road to identifying competencies in veterinary education. The authors established what was referred to as the Veterinary Professional, or VetPro framework (13).

The VetPro Framework was established in consultation with practicing veterinarians, and later validated internationally among practicing veterinarians. Veterinarians with clinical experience across many species groups were consulted in order to identify the core competency domains necessary for the practice of veterinary medicine across all typical practice settings. A total of seven competency domains were identified and defined. These included communication, personal development, collaboration, entrepreneurship, veterinary expertise, health and welfare, and scholarship (13). After the initial work that identified the competency domains was conducted in The Netherlands, an international survey was conducted to explore whether there was international consensus on the competency domains. Veterinarians in The Netherlands, Spain, Norway, United States, South Africa, Switzerland, Canada, United Kingdom, Malaysia, and Australia were included. While there was overall agreement with the importance of veterinary expertise as a competency domain, there were some differences in the importance of the other competency domains, although all were considered important to a degree (14). 
In 2015, the Competency-Based Veterinary Education (CBVE) Working Group was established to develop an internationally shared framework and assessment tools for use in veterinary education. The goal was to form a unified and comprehensive competency framework that reflects expectations of newly graduated veterinarians, guides learner assessment, and promotes targeted curricular outcomes assessment (15). Likewise, it was the intent that this framework would guide veterinary educational institutions in implementing competency-based methods of instruction. Nine competency domains were established as well as eight core Entrustable Professional Activities (EPAs) linked to the framework (15). An EPA is defined as an essential task that an individual can be trusted to perform without direct supervision in a given health care context, once sufficient competence has been demonstrated (16). To help learners and assessors monitor progression toward competence, milestones are often established within each EPA. Milestones are defined as observable markers of an individual's ability along a developmental continuum (16). Milestones for these veterinary EPAs have also been developed (17). The core competency domains established for veterinary education include (15):

1. Clinical Reasoning and Decision-making

2. Individual Animal Care and Management

3. Animal Population Care and Management

4. Public Health

5. Communication

6. Collaboration

7. Professionalism and Professional Identity

8. Financial and Practice Management

9. Scholarship

The eight EPAs established include (18):

1. Gather a history, perform an examination, and create a prioritized differential diagnosis list

2. Develop a diagnostic plan and interpret results

3. Develop and implement a management/treatment plan

4. Recognize a patient requiring urgent or emergent care and initiate evaluation and management

5. Formulate relevant questions and retrieve evidence to advance care

6. Perform a common surgical procedure on a stable patient, including pre-operative and post-operative management

7. Perform general anesthesia and recovery of a stable patient including monitoring and support

8. Formulate recommendations for preventive healthcare.

\section{Clinical Workplace-Based Assessments}

As is evident from the list of EPAs, the clinical workplace is the environment generally where demonstration of progress and assessment of entrustability can occur. EPAs typically span across multiple competency domains. The milestones provide a shared mental model for how learners are expected to developmentally progress throughout the program in providing a roadmap to building competence. Given that each EPA spans multiple competency domains, then a variety of assessment tools can be useful. Traditionally, in-training evaluation report scales (ITERs) have been used in veterinary education (19). They are usually completed by clinical preceptors who observe students throughout a clinical rotation. These rotations can span various time periods with two-to-four-week rotations common across veterinary institutions and are usually completed once at the end of the rotation. Rather than giving feedback about a particular moment in time, or a unique clinical encounter, they provide an assessment of the overall performance over the entire clinical rotation typically using a Likert-scale type of tool. They can encompass areas such as knowledge, clinical skills, interpersonal skills, and professionalism (see Figure 1). They are sometimes customized to fit the clinical environment where they are being used by clinical preceptors and faculty (20). Often, they require training and calibration of evaluators. For institutions that frequently use a large number of clinical preceptors and evaluators this can become problematic. Even when time is taken for calibration and instruction consistency of use can still be lacking as they are sometimes recognized by learners as "staff-dependent" (21). Variability between assessors, however, can be seen as providing valuable formative feedback for learners. Still, as EPAs are established in veterinary education the search for psychometrically sound and useful assessment tools will progress. Importantly, whatever tools are used need to not only assess entrustability, but also to provide formative feedback that can be used to guide learner progress, especially in areas like professionalism that remain somewhat ambiguous or subjective to many learners. As others have previously noted, clinical workplace-based assessments and entrustment scales can provide formative information and feedback to learners and help to determine when learners are able to engage in independent clinical practice (19). This formative process is essential to building competence and entrustability.

Clinical workplace-based assessments can be used strategically to steer learning toward desired outcomes, especially when formative timely feedback is provided during and after the assessment event (22). The form of the feedback can be both verbal and written. In addition to ITERs, there are other methods that have shown positive impacts on learning, including the Mini Clinical Evaluation Exercise (Mini-CEX) and the Direct Observation of Procedural Skills (DOPS) (23).

The Mini-CEX is used to evaluate learners while performing clinical tasks that often require demonstration of multiple skills simultaneously, such as taking a history while performing a physical examination of a patient. The core purpose is to provide structured feedback based on observed performance (22). Likert scaled scoring forms are often used that provide feedback not only on skills, such as physical examination, but also on communication and professionalism.

The DOPS assessment method focuses on evaluating procedural skills performed in the clinical workplace. Learners are provided a list of commonly performed procedures for which they are expected to demonstrate proficiency, and that are assessed by multiple clinicians on numerous occasions during an educational period of time (22). As with the Mini-CEX, the main goal of the DOPS is the provision of structured feedback based on observation of performance rather than on a simple completion 


\section{WesternU ITER for $4^{\text {th }}$ year clinical rotations (5-point Likert Scale)}

\begin{tabular}{|l|}
\hline KNOWLEDGE \\
\hline Exhibits core knowledge of basic sciences \\
\hline Exhibits basic knowledge of clinical material pertinent to rotation \\
\hline Is able to apply knowledge to clinical case management \\
\hline Creates relevant differential diagnoses \\
\hline Formulates appropriate therapeutic plans \\
\hline Locates and utilizes new information to supplement case management (i.e. primary literature) \\
\hline CLINCAL SKILLS \\
\hline Gathers appropriate history \\
\hline Performs thorough, technically accurate physical exam \\
\hline Accurately records information (quality of SOAP notes, record keeping) \\
\hline Displays suitable animal handling skills \\
\hline Demonstrates adequate technical ability \\
\hline Demonstrates good analytical skills, interpretation of findings and diagnostic procedures \\
\hline Demonstrates ability to critically appraise resources pertinent to case management \\
\hline Offers adequate quality of patient care \\
\hline INTERPERSONAL SKILLS \\
\hline Demonstrates effective verbal communication skills with clients and team members \\
\hline Presents written information in an accurate, logical, and organized manner \\
\hline Demonstrates compassion \\
\hline PROFESSIONALISM \\
\hline Is reliable, responsible and punctual \\
\hline Displays motivation to learn, enthusiasm and perseverance \\
\hline Is able to relate to clients, staff and peers \\
\hline Is honest, trustworthy; exhibits appropriate ethical and professional conduct \\
\hline
\end{tabular}

FIGURE 1 | Western University of Health Sciences ITER used during 4th year clinical rotations. log of procedures performed (22). The goal is to improve skills while performing specific procedures with the emphasis on the procedures themselves without much feedback given for competencies such as communication or professionalism.

While these three assessment and feedback instruments might sound similar, they do provide different information to learners. Take for instance a clinical scenario wherein a learner must successfully perform venipuncture on a dog after taking a history from the client and performing a physical examination. It is possible that proficiency could be marked as below expectations, meets expectations, or even exceeds expectations for the exact same clinical encounter based on the assessment tool used. Imagine that a veterinary student is given that specific task; to take a clinical history from a client, perform a physical examination, and to successfully draw blood from the patient, then divide the blood sample into the appropriate diagnostic collection tubes. During the encounter, the student only asks closed-ended questions and fails to elicit an important detail about the animal's clinical history. The student performs the physical exam adequately and note the appropriate abnormalities both verbally and in writing afterwards. During the venipuncture the student fails to communicate with the veterinary technician the proper restraint technique required and the client, who is standing close-by, gets bitten by their own dog. The learner, however, manages to collect the venipuncture sample and divides the sample appropriately into red-top and lavender-top tubes. If safety issues are incorporated into an ITER it would likely capture the obvious safety issue with improper restraint and injury to the client, resulting in a below-expectation score for the encounter even though the evaluation is a composite of the entire rotation because it would likely stand out in the preceptor's mind as a concern. A DOPS, however, would likely only note the successful collection and dividing of the blood samples, rating the event as meets or exceeds expectations. A Mini-CEX would provide feedback on the technical skills, communication, and collaboration with the technician, with ratings denoted as mixed results. Therefore, the tools used in the clinical workplace can make a profound difference in the focus of the feedback provided. This is important to recognize as EPAs span multiple competency domains and invites the discussion about whether multiple unique tools should be used when evaluating learning and providing formative feedback.

It has been proposed by veterinary educators and researchers to restructure veterinary education with EPAs (24). There is evidence that students are able to gradually gain experience in EPAs with various members of the clinical team (25). Using EPAs in the clinical workplace is a viable way to recognize student work and guide the development and progression of learners across the continuum of the various principles underlying them (26). Yet, whether in medical, veterinary, or other healthcare professions education, some of the competency domains can be more challenging to assess. Communication, collaboration, and professionalism are contextual and challenging to merely affirm that a learner was effective. Professionalism is a competency that some will say, "they know it when they see it," yet can be difficult to describe let alone create a scoring rubric. The IPA is a validated tool that is used by various members of the clinical team and provides formative feedback and evaluates various aspects of professionalism that are not readily captured by other evaluation tools. It has been used in veterinary education in both classroom and clinical settings to assess behaviors associated with professionalism in an interprofessional context. In the context of EPAs, the IPA could be used to help clinical educators and evaluators provide both formative and summative feedback to learners in domains difficult to assess with traditional tools. It could be used alongside these traditional tools by emphasizing areas that are typically missed with them, thereby providing a more holistic view and approach to clinical assessment. The real danger of not having specific tools to measure specific elements of EPAs is that some competencies may be merely passed over when tools emphasize only the more tangible hands-on skills. Worse still, learners may be given a "pass" on professionalism skills when they are condensed into other clinical skills performed at the time. The development of the IPA began with establishing a formal definition of interprofessional professionalism, which is stated as, "the consistent demonstration of core values evidenced by professionals working together, 
Interprofessional Professionalism Assessment (IPA) (5-point Likert Scale)

\section{COMMUNICATION}

Works with members of other health professions to coordinate communication with patients/clients and family members

Demonstrates active listening with members of other health professions

Communicates respectfully with members of other health professions

Communicates with members of other health professions in a way they can understand, without using professionspecific jargon

Responds to questions posed by members of other health professions in a manner that meets the needs of the requester

RESPECT

Demonstrates confidence, without arrogance, while working with members of other health professions

Recognizes that other health professions may have their distinct cultures and values, and shows respect for these

Respects the contributions and expertise of members of other health professions

Seeks to understand the roles and responsibilities of members of other health professions as related to care

Determines patient care roles and responsibilities in a respectful manner with members of other health professions

\section{ALTRUISM AND CARING}

Offers to help members of other health professions when caring for patients

Demonstrates empathy for members of other health professions

Models for other health professionals compassion towards patients/clients, families and caregivers

Places patient/client needs above own needs and those of other health professionals

\section{EXCELLENCE}

Coordinates with other health professions and the patient/client, family and caregivers to produce an optimal plan of care

Reviews all relevant documentation from other health care professions prior to making recommendations to plan of care

Contributes to decisions about patient care regardless of hierarchy/profession-based boundaries

Works with members of other health professions to assure continuity of care for patients

ETHICS

Interacts with members of other health professions in an honest and trustworthy manner

Works collaboratively with members of other health professions to resolve conflicts that arise in the context of caring for patients/clients

Discusses with members of other health professions any ethical implications of healthcare decisions

Reports or addresses unprofessional and unethical behaviors when working with members of other health professions ACCOUNTABILITY

Engages with members of other health professions in quality assurance/improvement activities

Seeks clarification from members of other health professions about unclear information

Accepts consequences for his or her actions without redirecting blame to members of other health professions

Works with members of other health professions to identify and address errors and potential errors in the delivery of care

FIGURE 2 | Interprofessional Professionalism Assessment (IPA). 
aspiring to, and wisely applying principles of altruism and caring, excellence, ethics, respect, communication, and accountability to achieve optimal health and wellness of individuals and communities" $(27,28)$.

\section{Interprofessional Professional Assessment (IPA)}

The IPA was created over a 9-year period through extensive development and pilot testing by the Interprofessional Professionalism Collaborative (IPC), a national organization with representatives currently from 12 entry-level health professions and the National Board of Medical Examiners. The IPA instrument is a 26 -item observational rating tool used by faculty and preceptors to assess learners' professionalism when working with members of other health professions. The tool was piloted at the end of a practice experience (e.g., rotation) in environments where interprofessional, collaborative care of patients was conducted. The psychometric properties of the IPA were tested with preceptors from 10 different health professions, including veterinary medicine, in seeking to support its generalizability. Psychometric results demonstrate aspects of IPA reliability and validity, and its use across multiple health professions and in various practice sites (27-29).

The development of the IPA was comprehensive and wellexecuted from 2006 to 2015 over three phases: (1) Construct development and generation of observable behaviors and response scales (27); (2) Content expert review and cognitive interviews with typical raters $(27,28)$; and (3) a 2-year pilot study (29). The process began with a literature review, construct definition, and the organization of 200 potential behaviors into categories by the members of the IPC. The number of behaviors was reduced to 43 after the IPC applied explicit inclusion criteria (e.g., behaviors that are positively oriented and observable in practice, applicable across multiple professions, not redundant). Members of the IPC then made national and international presentations about the tool, documented oral feedback from audience members, and collected follow-up online survey feedback from 205 individuals representing 11 professions. This feedback led to the formatting of a 39-item instrument, which was then reviewed by a panel of 23 content expert reviewers from the U.S. and Canada. The panel responded to structured survey questions about the tool's content, fit of 39 behavioral items within and across six domains, overall organization, format, and length (28).

Twenty-four preceptors, two from each of the 12 IPC member health professions representing "typical" preceptors that would use the tool, were involved in two rounds of cognitive interviews. Based on their feedback, the IPA was reduced to 26 items categorized into six competency domains which included (see Figure 2):
1. Communication
2. Respect
3. Altruism and Caring
4. Excellence

5. Ethics

6. Accountability

The 26 behavioral items are constructed on a 5-point, Likerttype scale with the following anchors: $1=$ "strongly disagree," $2=$ "disagree," $3=$ "neutral," $4=$ "agree," and $5=$ "strongly agree." For each behavior there is also the response option, "no opportunity to observe." The two qualitative items provide space for raters to comment on the "overall strengths related to interprofessional professionalism" and "areas for improvement related to interprofessional professionalism” (28).

The 26-item instrument is the version of the IPA which was used in a large, multi-institution and multi-profession pilot study. A total of 67 academic institutions were invited to participate in the pilot; 30 agreed to do so (44.8\%). Using a key contact method, nearly 3,000 preceptors (estimated) were invited into the study; 376 agreed and 233 provided data (62\% of enrolled, $7.9 \%$ of potential population) (29).

Exploratory factor analysis (EFA) was conducted on preceptors' ratings of their learners assuming ordered categorical factor indicators. To determine the number of factors to retain, eigenvalues and measures of fit were examined. Prior to factor analysis, the extent of missing data for each of the IPA items was examined (i.e., an item either left blank or the respondent recorded N/O - No opportunity to observe in this environment). Internal consistency reliability of the factors suggested by the EFA was calculated using coefficient alpha. The initial EFA using 21 items (excluding five items with extensive missing data) suggested retaining four factors. With eigenvalues of $12.670,1.229,0.888$, and 0.787 , the four factors together accounted for $86.5 \%$ of the variance in the set of variables, and the fit indices indicated good model fit (RMSEA $=0.064$, 90\% CI: 0.055-0.078; CFI = 0.991; SRMR = 0.027). The four factors loaded well on the following domains: Communication, Respect, Excellence, Altruism, and Caring. Internal reliability consistency coefficients were high (alpha >0.94) for each of the factors. Despite the psychometric results, and based on other considerations, the study authors decided to keep the 5 excluded items and the two other domains (Ethics and Accountability) in the final instrument (29).

During the pilot study, responding preceptors were also asked to complete two global items for each learner they evaluated: one was a global rating of the learner's interprofessional professionalism, and the other a global rating of the learner's overall performance on the practice experience. These ratings were made using a 5 -point Likert-type scale $(1=$ "poor," $5=$ "excellent"). Given the results of the factor analysis, items within each domain were averaged to create subscale scores and factor scores were also estimated from the final factor model. These scores were all positively and significantly correlated with the two global performance items described above $(29,30)$.

The Interprofessional Professionalism Collaborative (IPC) has a website (http://www.interprofessionalprofessionalism.org/ assessment.html) from which a PDF of the IPA instrument can be downloaded for use. The IPC website also has a toolkit which provides training videos for users (e.g., students, faculty, preceptors) and narrative written and recorded scenarios to 
support training in the use and application of the IPA in their practices and programs.

\section{DISCUSSION}

The introduction of competency-based education in veterinary medicine requires not only a change in how we educate, but perhaps more importantly a change in how we assess learners. Historically, assessments in the final clinical years of veterinary education have been less formalized than written assessments in the pre-clinical years. But with an emphasis on competence and entrustability, assessment in the clinical years is perhaps more important than traditional multiple-choice types of exams during the pre-clinical years. While there has been tremendous progress in defining competence, and describing competence-based education, many still struggle with methods of assessment that are valid and reliable. Workplace-based assessments have been studied and examined more extensively in medical education than in veterinary education. Yet, one must be careful to accept validation only in medical education and expecting similar applicability to veterinary education. Therefore, there is extreme importance in validating instruments in the veterinary setting and amending those with re-validation, as applicable.

The IPA is an example of an assessment tool that has been studied and validated in the veterinary setting (29). As an interprofessional assessment tool, it is important to note that interactions with other health professions are not frequent in veterinary practice. A study examining the frequency of interactions between veterinarians and other healthcare providers found that interactions with pharmacists was the most common interprofessional interaction (31). In the study and validation of the IPA, a total of 10 professions, including veterinary medicine, were included (29). The ratings on the IPA for veterinary interprofessional interactions were scored similarly to interactions that did not include a veterinary component. Veterinary students at Western University of Health Sciences, College of Veterinary Medicine (WesternU CVM) were participants in the IPA validation study. It is important to note that the WesternU CVM utilizes a distributive model of clinical education as opposed to a standing university teaching clinic. As such, many clinical rotations occur in the greater Los Angeles metropolitan area where interactions with other health professions were most likely to be with pharmacists and in some instances with physical therapists in animal rehabilitation settings.

While the design and validation of the IPA tool was not originally intended for intra-professional interactions, the IPA tool does have applicability when evaluating interactions with veterinary technicians, animal assistants, farriers, and others within the broader field of veterinary practice. A major impetus for interprofessional education and collaborative practice efforts has been to improve patient outcomes and safety (32-34). The Institute of Medicine reports have emphasized how patients are harmed when communication and collaboration fail in healthcare. Failures in communication and collaboration have been reported as preventable causes of death and injury in healthcare settings (32). While it is generally unclear if the same deleterious effects occur in the veterinary setting when communication and collaboration fail, there are indications that the patterns may be similar (35). Further, substantial barriers may exist for reporting significant events in veterinary practice (36). The reasons for under-reporting are often attributes and behaviors that are captured in the IPA tool, specifically under the competency heading of accountability. There is a clear need to better assess professionalism behaviors in veterinary learners not only to improve the quality and safety of care and thus patient outcomes, but also to promote effective collaboration within the veterinary team, and to improve relationship-centered care with veterinary clients.

Increasingly, veterinarians are being employed outside of the historic, traditional veterinary practice owned by one or a few of the veterinarians working there. Corporate veterinary practices are expanding across the entire US. Additionally, specialty practices are expanding in both the companion animal and production animal veterinary markets. The idea of the veterinary team consisting of a group of people working under the same roof, with similar working hours is being replaced by primary care and specialty care teams working across geographic locations in multiple practices operating 24 hours a day and 365 days a week. Teamwork skills have never been needed more in veterinary medicine in order to maintain the quality of care and ensure the safety of patients and the public. Veterinary careers also are expanding in research and biotechnology organizations, in public health sectors, in non-profit organizations, and in government. With these expansions the veterinary team is changing. Clinical veterinary teams may include specialists working under the umbrella of a corporate veterinary practice with locations spanning several cities or states across the country. New veterinary specialty organizations are in the works, with further expansions of specialty care being defined. Outside of clinical practice, veterinary teams might include epidemiologists and environmental scientists working in public health, policymakers and lawmakers working in government, and engineers and computer scientists working in industry to name a few, where professionalism takes on a broader perspective and includes new stakeholders. The need to train veterinarians and veterinary students in professionalism behaviors and effective teamwork practices and skills has never been greater. Along with the need to educate comes the even greater need to reliably assess such learning. Not only do professionalism behaviors need to be consistently defined, they must also be reliably assessed wherever veterinary teams are working. And this is reflected in the competency domains identified and defined by work done by the CBVE.

The IPA presents many opportunities to discuss attributes and behaviors associated with professionalism with veterinary students, throughout the professional veterinary educational program. At WesternU it has been used successfully with preclinical years students in formalized interprofessional education courses that include veterinary students. With students in the pre-clinical years, it is not possible to use the tool in the workplace-based setting. Instead, filmed interprofessional 
interactions (scripted) are used. To begin, information is provided to students about the IPA tool and initially introduced through an assigned reading, then reviewed in the classroom setting. Application of the IPA is demonstrated by showing a filmed interprofessional interaction. Each student is assigned a clinician (actor) to review using the IPA tool. They first watch the filmed interaction and are instructed to take notes on what they observe. Next, they are provided portions of the IPA tool to review and instructed to watch the same video a second time. This time they are asked to complete the IPA portions applicable to the scene. Students complete this activity as individuals without first comparing their reviews. Later they compare their evaluations with those of their peers and discuss how they arrived at their scores, noting specific behaviors that they observed that support the ratings. Finally, students discuss how and why multiple people watching the same interactions might score them differently. The scripted scenes used were carefully chosen because the words that the actors use do not always reflect the intentions in their actions. It provides rich opportunities for students to discuss the power of both verbal and non-verbal communication, and the implications when collaboration and communication fail. During the debrief of the activity, the discussion often includes hierarchical issues in healthcare that impact how individuals within certain professions behave and misbehave in the clinical environment. It is hoped that through these classroom activities, students are sensitized to notice communication successes and failures when in the clinical workplace environments in subsequent years of the curriculum.

\section{CURRENT AND FUTURE WORK}

While the IPA was developed using the traditional definition of interprofessional education which includes learners from different healthcare professions learning with, from, and about one another in order to improve collaboration and the quality of care, it has value when applied to the intraprofessional veterinary team (37). The typical clinical veterinary healthcare team includes veterinarians, veterinary technicians or nurses, animal assistants, and others depending on the practice environment. With the expansion of specialty practices, especially in major urban centers, the veterinary team can include veterinary specialists and primary care clinicians working collaboratively with the same patients but in different practice settings. Often these collaborations occur across distant practice settings and not within the same hospital, as is common within human healthcare hospitals and clinics. When communication fails, collaboration is not promoted, and respect is absent or lacking, there may be a failure to deliver optimal care for a given situation, or harm may come to the veterinary patient $(35,38)$. In a study examining errors in veterinary practices, many of the root causes were aligned with communication failures (both verbal and health record omissions), and failures in team functioning (39). These errors often involved failures in collaboration among veterinarians, veterinary nurses, and receptionists. Failures in communication may ultimately become a potential legal liability; indeed, failures in communication and deficiencies in interpersonal skills were linked to complaints against veterinarians filed with state veterinary boards (40). Additionally, the presence or absence of these skills appear to impact professional well-being and satisfaction. A primary reason for veterinary nurses leaving the profession was found to be "lack of respect and recognition from veterinarians" (39).

A core tenet of interprofessionalism involves placing the patient and client at the center of the healthcare team. In the veterinary setting this translates to client-centeredness. The veterinary client hierarchy of needs suggested by Hughes et al. (41) focuses on working in partnership with clients, and emphasizes the importance of communication skills, professionalism, clinical problem-solving, and the animal's welfare in achieving excellence. These aspects are captured well with the IPA, and therefore it is also applicable to apply its use while evaluating veterinary students' interactions with clients.

Interprofessional learning within the veterinary healthcare team has been successfully piloted and demonstrated to change attitudes, overcome misconceptions about the professions and promote the importance of communication between veterinarians and veterinary nurses (42). Veterinary students also participate in traditional interprofessional education programs, and benefit from learning with, from and about students in the human healthcare professions (43). The IPA has been validated and used in the veterinary education setting and can be applied and used in the education of both veterinarians and veterinary technicians/nurses. Therefore, more work could be done evaluating its merits within existing veterinary teams, and while educating both veterinary students and veterinary technicians, whose educational programs tend to be unconnected and isolated even though they work very closely together after graduation.

In addition to traditional veterinary teams working in typical practice locations, the veterinary team also includes those working in academia, government, public health, biotechnology, and industry among others. The IPA has applicability in these teams as well with very minor modifications such as replacing "patient" with "stakeholder" for example. The importance of professionalism does not stop at clinical locations or at the cage-side. Future work might include these expanded veterinary roles working in teams that are indeed interprofessional, but not necessarily with the professions that usually come to mind when thinking only of the clinical setting.

Other areas of future work could be in comparing use and applicability in urban vs. rural settings, where the needs of clients and stakeholders might be perceived differently. While the needs might not be that different in actuality, the culture within an organization may impact how professionalism behaviors are demonstrated and addressed. Therefore, examining the IPA tool in various urban and rural locations might help to provide important data.

Lastly, improving diversity within the veterinary profession and within veterinary teams is important and a focus of national and international attention. While the IPA tool was investigated in a large-scale pilot it was not examined for applicability of use with various ethnic groups, races, 
ages, gender, or gender identity. Designing studies to look at its applicability across these and other social constructs is warranted.

\section{AUTHOR CONTRIBUTIONS}

All authors listed have made a substantial, direct and intellectual contribution to the work, and approved it for publication.

\section{REFERENCES}

1. Tyler RW. Basic Principles of Curriculum and Instruction. Chicago: The University of Chicago Press (1949) (Paperback edition 1969).

2. ten Cate O, Scheele F. Viewpoint. Competency-based postgraduate training: can we bridge the gap between theory and clinical practice? Acad Med. (2007) 82:542-7. doi: 10.1097/ACM.0b013e31805559c7

3. Adams NE. Bloom's taxonomy of cognitive learning objectives. J Med Libr Assoc. (2015) 103:152-3. doi: 10.3163/1536-5050.103.3.010

4. Frye AW, Hemmer PA. Program evaluation models and related theories: AMEE Guide No. 67. Med Teach. (2012). 34:e28899. doi: 10.3109/0142159X.2012.668637

5. McGaghie WC, Miller GE, Sajid AW, Telder TV. Competency-Based Curriculum Development in Medical Education. Geneva: World Health Organization (1978).

6. ten Cate O. Competency-based postgraduate medical education: past, present and future. GMS J Med Educ. (2017) 34:Doc69. doi: 10.3205/zma001146

7. Frank JR, Danoff D. The CanMEDS initiative: implementing an outcomesbased framework of physician competencies. Med Teach. (2007) 29:6427. doi: 10.1080/01421590701746983

8. ten Cate O, Chen HC, Hoff RG, Peters H, Bok HGJ, van der Schaaf M. Curriculum development for the workplace using Entrustable Professional Activities (EPAs): AMEE Guide No. 99. Med Teach. (2015) 37:9831002. doi: 10.3109/0142159X.2015.1060308

9. Holmes WM, Scaffa ME. An exploratory study of competencies for emerging practice in occupational therapy. J Allied Health. (2009) 38:81-90.

10. Jung B, Shimmell L, Stewart D, Gatti L, Venasse K, Plaisant L, et al. Competency-based education: a survey study of international occupational therapy educational programmes. World Fed. Occup. Phys. Ther. Bull. (2015) 71:53-8. doi: 10.1179/1447382815Z.0000000009

11. Chesbro SB, Jensen GM, Boissonnault WG. Entrustable professional activities as a framework for continued professional competence: is now the time? Phys Therapy. (2018) 98:3-7. doi: 10.1093/ptj/pzx100

12. Hodges AL, Konicki AJ, Talley MH, Bordelon CJ, Holland AC, Galin FS. Competency-based education in transitioning nurse practitioner students from education into practice. J Am Assoc Nurse Pract. (2019) 31:67582. doi: 10.1097/JXX.0000000000000327

13. Bok HGJ, Jaarsma DADC, Teunissen PW, van der Vleuten CPM, van Beukelen P. Development and validation of a competency framework for veterinarians. J Vet Med Educ. (2011) 38:262-9. doi: 10.3138/jvme.38.3.262

14. Bok HGJ, Teunissen PW, Boerboom TBB, Rhind SM, Baillie S, Tegzes JH, et al. International survey of veterinarians to assess the importance of competenices in professional practice and education. J Am Vet Med Assoc. (2014) 245:90613. doi: 10.2460/javma.245.8.906

15. Matthew SM, Bok HGJ, Chaney KP, Read EK, Hodgson JL, Rush $\mathrm{BR}$, et al. Collaborative development of a shared framework for competency-based veterinary education. J Vet Med Educ. (2020) 47:579-93. doi: 10.3138/jvme.2019-0082

16. Englander R, Frank JR, Carraccio C, Sherbino J, Ross S, Snell L, on behalf of the ICBME Collaborators. Toward a shared language for competency-based medical education. Med Teach. (2017) 39:5827. doi: 10.1080/0142159X.2017.1315066

17. AAVMC Working Group on Competency-Based Veterinary Education, Molgaard LK, Hodgson JL, Bok HGJ, Chaney KP, Ilkiw JE, et al. Competency-Based Veterinary Education: Part 1 - CBVE Framework.

\section{ACKNOWLEDGMENTS}

We acknowledge with appreciation all those who have participated as members of the Interprofessional Professionalism Collaborative, and the participating health professions organizations, who have contributed to the development and validation of the Interprofessional Professionalism Assessment.

Washington, DC: Association of American Veterinary Medical Colleges (2018).

18. Molgaard LK, Chaney KP, Bok HGJ, Read EK, Hodgson JL, Salisbury SK, et al. Development of core entrustable professional activities linked to a competency-based veterinary education framework. Med Teach. (2019) 41:1404-10. doi: 10.1080/0142159X.2019.1643834

19. Read EK, Brown A, Maxey C, Hecker KG. Comparing entrustment and competence: an exploratory look at performance-relevant information in the final year of a veterinary program. J Vet Med Educ. (2020). doi: 10.3138/jvme-2019-0128. [Epub ahead of print].

20. Weijs CA, Coe JB, Hecker KG. Final-year students' and clinical instructors' experience of workplace-based assessments used in a smallanimal primary-veterinary-care clinical rotation. J Vet Med Educ. (2015) 42:382-92. doi: 10.3138/jvme.1214-123R1

21. Ginsburg S, van der Vleuten CPM, Eva KW, Lingard L. Cracking the code: residents' interpretations of written assessment comments. Med Educ. (2017) 51:401-10. doi: 10.1111/medu.13158

22. Norcini J, Burch V. Workplace-based assessment as an educational tool: AMEE Guide No. 31. Med Teach. (2007) 29:855-71. doi: 10.1080/01421590701775453

23. Lorwald AC, Lahner F-M, Nouns ZM, Berendonk C, Norcini J, Greif R, et al. The educational impact of Mini-Clinical Evaluation Exercise (Mini-CEX) and Direct Observation of Procedural Skills (DOPS) and its association with implementation: a systematic review and meta-analysis. PLoS ONE. (2018) 13:30198009. doi: 10.1371/journal.pone.0198009

24. Duijn C, Bok H, ten Cate O, Kremer W. Qualified but not yet fully competent: perceptions of recent veterinary graduates on their day-one skills. Vet Record. (2019) 186:216. doi: 10.1136/vr.105329

25. Favier RP, ten Cate O, Duijn C, Bok HGJ. Bridging the gap between undergraduate veterinary training and veterinary practice with entrustable professional activities. J Vet Med Educ. (2021) 48:136-8. doi: 10.3138/jvme.2019-0051

26. Chen HC, van den Broek WES, ten Cate O. The case for use of entrustable professional activities in undergraduate medical education. Acad Med. (2015) 90:431-6. doi: 10.1097/ACM.0000000000000586

27. Holtman MC, Frost JS, Hammer DP, McGuinn K. Nunez L. Interprofessional professionalism: linking professionalism and interprofessional care. J Interprof Care. (2011) 25:383-5. doi: 10.3109/13561820.2011.588350

28. Hammer DP, Frost J, Anderson MB, Brunson WD, Grus C, et al. Defining and measuring construct of interprofessional professionalism. J Allied Health. (2012) 41:e49-53.

29. Frost JS, Hammer DP, Nunez LM, Adams JL, Chesluk B, Grus C, et al. The intersection of professionalism and interprofessional care: development and initial testing of the interprofessional professionalism assessment (IPA). J Interprof Care. (2019) 33:102-15. doi: 10.1080/13561820.2018.1515733

30. Schmitz CC. Interprofessional Professionalism Assessment. National Center for Interprofessional Practice and Education: Assessment and Evaluation (2018). Available online at: https://nexusipe.org/advancing/assessment-evaluation/ interprofessional-professionalism-assessment-ipa (accessed March 23, 2021).

31. Root Kustritz MV, Molgaard LK, Tegzes JH. Frequency of interactions between veterinarians and other professionals to guide interprofessional education. J Vet Med Educ. (2013) 40:370-7. doi: 10.3138/jvme.0413-065R1

32. Institute of Medicine (US) Committee on Quality of Health Care in America, Kohn LT, Corrigan JN, Donaldson MS, editors. To Err is Human: Building a Safer Health System. Washington, DC: National Academic Press (US) (2000). 
33. Institute of Medicine (US) Committee on Quality of Health Care in America. Crossing the Quality Chasm: A New Health System for the 21st Century. Washington, DC: National Academic Press (US) (2001).

34. Institute of Medicine (US) Committee on the Health Professions Education Summit, Greiner AC, Knebel E, editors. Health Professions Education: A Bridge to Quality. Washington, DC: National Academic Press (US) (2003).

35. Oxtoby C, Ferguson E, White K, Mossop L. We need to talk about error: causes and types of error in veterinary practice. Vet Record. (2015) 177:438. doi: 10.1136/vr.103331

36. Oxtoby C, Mossop L. Blame and shame in the veterinary profession: barriers and facilitators to reporting significant events. Vet Record. (2019) 184:501. doi: 10.1136/vr.105137

37. Barr H. Interprofessional education - today, yesterday and tomorrow. Higher education academy, learning \& teaching support network for health sciences \& practice, occasional paper 1. UK Centre for the Advancement of Interprofessional Education (CAIPE) (2002).

38. Kinnison T, Guile D, May SA. Errors in veterinary practice: preliminary lessons for building better veterinary teams. Vet Record. (2015) 177:492. doi: 10.1136/vr.103327

39. Kinnison T, May SA. Veterinary nurses and interprofessional working. Vet Record. (2015) 177:345. doi: 10.1136/vr.h5218

40. Russel RL. Preparing veterinary students with the interactive skills to effectively work with clients and staff. J Vet Med Educ. (1994) 21:40-3.
41. Hughes K, Rhind SM, Mossop L, Cobb K, Morley E, Kerrin M, et al. 'Care about my animal, know your stuff and take me seriously': United Kingdom and Australian clients' views on the capabilities most important in their veterinarians. Vet Record. (2018) 183:534. doi: 10.1136/vr. 104987

42. Kinnison T, Lumbis R, Orpet H, Welsh P, Gregory S, Baillie S. Piloting interprofessional education interventions with veterinary and veterinary nursing students. J Vet Med Educ. (2011) 38:311-8. doi: 10.3138/jvme.38. 3.311

43. Tegzes JH. Chapter 7. Teaching interprofessionalism. In: Hodgson JL, Pelzer JM, editors. Veterinary Medical Education: A Practical Guide. Hoboken, NJ: Wiley-Blackwell (2017). p. 101-15.

Conflict of Interest: The authors declare that the research was conducted in the absence of any commercial or financial relationships that could be construed as a potential conflict of interest.

Copyright $\odot 2021$ Tegzes and Frost. This is an open-access article distributed under the terms of the Creative Commons Attribution License (CC BY). The use, distribution or reproduction in other forums is permitted, provided the original author(s) and the copyright owner(s) are credited and that the original publication in this journal is cited, in accordance with accepted academic practice. No use, distribution or reproduction is permitted which does not comply with these terms. 